\title{
Review on Effect of Powder Mixed Dielectric on Material Removal Rate in Electric Discharge Machining
}

\author{
M. S. Bembde ${ }^{1}$, J. K. Sawale ${ }^{2}$ \\ ${ }^{1}$ P.G .Student, Department of Mechanical Engineering, M.G.M"es College of Engineering, Nanded, Maharashtra, India \\ ${ }^{2}$ Professor, Department of Mechanical Engineering, MGM. “s College of Engineering, Nanded, Maharashtra, India
}

\begin{abstract}
Powder mixed electric discharge machining (PMEDM) is a recent innovation for enhancing the capabilities of electrical discharge machining process. The objective of present study is to realize the potential of silicon powder as additive in enhancing machining capabilities of PMEDM. Taguchi methodology has been adopted to plan and analyze the experimental results. L16 Orthogonal Array has been selected to conduct experiments. Peak current, Pulse on time, pulse off time, voltage, and concentration of fine silicon powder added into the dielectric fluid were chosen as input process variables to study performance in terms of material removal rate. The ANOVA analysis identifies the most important parameters to maximize material removal rate.
\end{abstract}

Keywords: Electrical discharge machining, Machining rate, Spark gap, Silicon powder concentration, Taguchi methodology.

\section{Introduction}

Electric discharge machining is a thermo-electric nontraditional machining process. Material is removed from the work piece through localized melting and vaporization of material. Electric sparks are generated between two electrodes when the electrodes are held at a small distance from each other in a dielectric medium and a high potential difference is applied across them. Localized regions of high temperatures are formed due to the sparks occurring between the two electrode surfaces. Work piece material in this localized zone melts and vaporizes. Most of the molten and vaporized material is carried away from the inter-electrode gap by the dielectric flow in the form of debris particles. To prevent excessive heating, electric power is supplied in the form of short pulses. Spark occurs wherever the gap between the tool and the work piece surface is smallest. After material is removed due to a spark, this gap increases and the location of the next spark shifts to a different point on the work piece surface. In this way several sparks occur at various locations over the entire surface of the work piece corresponding to the work piece-tool gap. Because of the material removal due to sparks, after some time a uniform gap distance is formed throughout the gap between the tool and the work piece.

\section{Literature Review}

\subsection{INTRODUCTION TO PAST RESEARCH}

Following are the researchers, who have worked in the area of silicon powder mixed dielectric EDM process for high carbon steel.

H.K.Kansal and Pradeep kumar [1] In this paper, the effect of silicon powder mixing into the dielectric fluid of EDM on machining characteristics of AISI D2 (a variant of high carbon high chrome) die steel has been studied. Six process parameters, namely peak current, pulse on time, pulse-off time, concentration of powder, gain, and nozzle flushing have been considered. The process performance is measured in terms of machining rate (MRR). The research outcome will identify the important parameters and their effect on MRR of AISI D2 in the presence of suspended silicon powder in a kerosene dielectric of EDM. The study indicated that all the selected parameters except nozzle flushing have a significant effect on the mean and variation in MRR ( $\mathrm{S} / \mathrm{N}$ ratio). Optimization to maximize MRR has also been undertaken using the Taguchi method. The ANOVA analysis indicates that the percentage contribution of peak current and powder concentration toward MRR is maximum among all the parameters. The confirmation runs showed that the setting of peak current at a high level (16 A), pulse-on time at a medium level $(100 \mu \mathrm{s})$, pulse-off time at a low level $(15 \mu \mathrm{s})$, powder concentration at a high level (4 g/l), and gain at a low level $(0.83 \mathrm{~mm} / \mathrm{s})$ produced optimum MRR from AISI D2 surfaces when machined by silicon powder mixed EDM.

M. Kiyak and O. Cakir [2], this paper is devoted to a study of the influences of EDM parameters on surface roughness for machining of 40CrMnNiMo864 tool steel (AISI P20) which is widely used in the production of plastic mold and die. The selected EDM parameters were pulsed current $(8$, 16 and $24 \mathrm{~A})$, pulse time $(2,3,4,6,12,24,48$ and $100 \mu \mathrm{s})$ and pulse pause time ( 2 and $3 \mu \mathrm{s}$ ). It was observed that surface roughness of work piece and electrode were influenced by pulsed current and pulse time, higher values of these parameters increased surface roughness. Lower current, lower pulse time and relatively higher pulse pause time produced a better surface finish.

Anirban Bhattacharya \& Ajay Batish \& Gurmail Singh \&V. K. Singla [3], the present study was undertaken to identify the appropriate parameter settings for rough and finish machined surface for EN31, H11, and high carbon high chromium (HCHCr) die steel materials in a powdermixed electric discharge machining process. The effect of seven different process variables along with some of their 


\section{International Journal of Science and Research (IJSR) \\ ISSN (Online): 2319-7064 \\ Index Copernicus Value (2013): 6.14 | Impact Factor (2014): 5.611}

interactions was evaluated using a dummy-treated experimental design and analysis of variance. Material removal rate (MRR), tool wear rate, and surface finish were measured after each trial and analyzed. The parameter settings for rough and finished machining operations were obtained. EN31 exhibited maximum MRR as compared to the other two materials at similar process settings. Copper $\mathrm{Cu}$ ) electrode with aluminum suspended in the dielectric maximized the MRR. Suspending powder in the dielectric resulted in surface modification. Graphite powder showed a lower MRR but improved the surface finish. $\mathrm{HCHCr}$ require higher current and pulse on settings for initiating a machining cut and works best in combination with tungsten$\mathrm{Cu}$ electrode and graphite powder for improved finish. The MRR for H11 is lower than EN31 but significantly higher than $\mathrm{HCHCr}$ under same process conditions.

Katsushi Furutani \& Hiromichi Sato \& Masayuki Suzuki [4], paper describes the influence of the discharge current and the pulse duration on the titanium carbide (TiC) deposition process by electrical discharge machining (EDM) with titanium (Ti) powder suspended in working oil. Although the influence of the electrical conditions for removal EDM has been investigated, the criteria for deposition have not been discussed. In the experiments, a 1$\mathrm{mm}$ copper rod was used for an electrode to prevent the flushing of working oil from the gap between the electrode and a work piece. Ti powder reacted with the cracked carbon from the working oil, then depositing a TiC layer on a work piece surface. A major criterion of the deposition or removal was the discharge energy over a pulse duration of $10 \mu \mathrm{s}$. A thickness of the $\mathrm{TiC}$ layer became the maximum at a certain discharge current and pulse duration. Larger discharge energy and power promoted the removal by heat and pressure caused by the discharge. The removal was classified further into two patterns; cracks were observed on the Ti-rich surface in removal pattern 1 and a workpiece was simply removed in removal pattern 2 . The maximum hardness of the deposition was $2000 \mathrm{Hv}$. The work piece about $10 \mu \mathrm{m}$ beneath its surface was also hardened because of the dispersion of $\mathrm{TiC}$. The machining conditions for the hardest deposition did not coincide with those for the highest one. Therefore, the discharge current and pulse duration should be optimized for the deposition.

Paulo Peças \& Elsa Henriques [5], The addition of powder particles to the electrical discharge machining (EDM) dielectric fluid modifies some process variables and creates the conditions to achieve a higher surface quality in large machined areas. This paper presents a new research work that aims to study the improvement in the polishing performance of conventional EDM when used with a powder-mixed-dielectric (PMDEDM). The analysis was carried out varying the silicon powder concentration and the flushing flow rate over a set of different processing areas and the effects in the final surface were evaluated. The evaluation was done by surface morphologic analysis and measured through some quality surface indicators. The results show the positive influence of the silicon powder in the reduction of crater dimensions, white-layer thickness and surface roughness. Moreover, it was demonstrated that an accurate control of the powder concentration and flushing flow is a requirement for achieving an improvement in the process polishing capability.

Promod K. Patowari \& Partha Saha \& Prasanta K. Mishra [6], The present study describes an experimental research on surface modification during electrical discharge machining (EDM) by depositing a hard layer over the work surface of C-40 grade plain carbon steel using specially prepared powder metallurgy compact tools. The investigated process parameters were composition, compaction pressure, sintering temperature, pulse on-time, peak-current setting, and duty factor. Measurements of deposited layer thickness, mass transfer rate, tool wear rate, surface roughness and micro hardness were undertaken on the EDM-ed specimens. Different studies like X-ray diffraction, optical microscopy, scanning electron microscopy, and energy dispersive X-ray spectroscopy were carried out to ascertain the characteristics of the deposited layer on the work surface. These analyses confirmed the presence of the tool materials in the work surface layer. At first, an L-16 orthogonal array was applied as Taguchi DOE technique and the ANOVA was done to study the effects of pertinent process parameters. An optimum condition was achieved using overall evaluation criteria. Later on, a detailed study was carried out to get a smooth and regular deposition of material. The characterization of the deposited layer is presented. The deposited layer with a wide range of average layer thickness of 3-785 $\mu \mathrm{m}$, enriched with tool materials (Wand $\mathrm{Cu}$ ) and with the formation of tungsten carbide, and having micro hardness of 9.81-12.75 $\mathrm{GPa}$ at the hardest zone was successfully deposited over the work surface.

KEESAM SHIN, DONGSUP CHUNG, and SUNGHAK LEE [7], the effects of consolidation temperature on the development of microstructure and resulting mechanical properties of $2 \mathrm{XXX}$ aluminum composites were studied in an effort to fabricate composites with enhanced properties. Type 2009 and 2124 aluminum composites reinforced with 15 pct $\mathrm{SiC}$ particulates were produced at four different consolidation temperatures, i.e., 560 7C, 580 7C, $6007 \mathrm{C}$, and $6207 \mathrm{C}$, followed by extrusion at $4507 \mathrm{C}$. The $2124 \mathrm{Al}-$ $\mathrm{SiCp}$ composites consolidated at $5607 \mathrm{C}$ showed the most homogeneous and the finest microstructures with the best mechanical properties, which were even better than the whisker-reinforced counterparts. All the results of the tensile tests, hardness tests, in situ scanning electron microscope (SEM) observations of the fracture process, and the apparent fracture toughness indicated that the prominent mechanical property improvement observed in the $2124 \mathrm{Al}-\mathrm{SiC} p$ was associated largely with the reduction of volume fraction of the detrimental coarse and brittle manganese-containing particles, as well as grain refinement. The detrimental manganese-containing particles that were routinely observed in the $2124 \mathrm{Al}-\mathrm{SiC}$ composites were very effectively refined by the reduction of consolidation temperature, and they rather contributed to the overall mechanical properties of the composites through Orowan-type strengthening and grain growth inhibition.

Y.-F. Tzeng and C.-Y. Lee [8], this paper presents the effects of various powder characteristics on the efficiency of electro discharge machining (EDM) SKD-11. The additives examined include aluminium $(\mathrm{Al})$, chromium $(\mathrm{Cr})$, copper $(\mathrm{Cu})$, and silicon carbide $(\mathrm{SiC})$ powders that have significant

\section{Volume 4 Issue 12, December 2015}




\section{International Journal of Science and Research (IJSR) \\ ISSN (Online): 2319-7064 \\ Index Copernicus Value (2013): 6.14 | Impact Factor (2014): 5.611}

differences in their thermo physical properties. The machining mechanism with the addition of the foreign particles, the tool wear rate (TWR), and the material removal rate (MRR) have been investigated. It was found experimentally that the particle concentration, the particle size, the particle density, the electrical resistivity, and the thermal conductivity of powders were important characteristics that significantly affected the machining performance in the EDM process. Proper addition of powders to the dielectric fluid increased the MRR and, thus, decreased the TWR. Under the same particle concentration experiments, the smallest suspended particle size led to the greatest MRR and, thus, the lowest TWR. Of the additives investigated, chromium powder produced the greatest MRR and the lowest TWR, whereas the process without foreign particles has the converse effects. The addition of copper powder to the dielectric fluid was found to make almost no difference to the pure kerosene EDM system.

Han-Ming Chow, Lieh-Dai Yang, Ching-Tien Lin, YuanFeng Chen [9], Micro-slit EDM process along with small discharge energy and $\mathrm{SiC}$ powder in pure water was investigated in this study. SiC powder was added to pure water as a working uid to verify the micro-slit EDM process performance. Results indicated that the addition of $\mathrm{SiC}$ powder would increase working fluid electrical conductivity, enlarge the electrode and work piece gap, and also extrude debris easily, therefore increasing the material removal rate. Furthermore, the use of $\mathrm{SiC}$ powder helped bridge the electrode and work piece gap and disperse discharge energy, thus creating two discrete discharging pulses from a single discharging period that could effectively disperse discharging energy into several increments. The discharging results could then generate a minor crater and debris since minor debris would ease gap exhaust and accelerate material removal rate. The minor crater could simultaneously refine the surface roughness.

Farhad Kolahan Mohammad Bironro [10], In this paper, mathematical models are proposed, using regression method, to model and analysis the effects of machining parameters on the machining characteristics in the PMEDM process. In this regard, the effects of four machining parameters (grain size of aluminum powder, concentration of the powder, discharge current and pulse on time) on the important process outputs, including metal removal rate and electrode wear rate, have been investigated. To model the machining process, different regression functions have been fitted to the experimental data. Then, using analysis of variance (ANOVA), the best and most fitted set of models are identified. In addition to influence of individual machining parameters, the interactions between these parameters are also investigated. Finally, a genetic algorithm procedure has been employed to optimize the process parameters for any set of desired outputs. The results show that the proposed solution procedure performs very well in solving such complicated and non-linear optimization problems.

Kuang-Yuan Kung \& Jenn-Tsong Horng \& Ko-Ta Chiang [11], In this article, a material removal rate (MRR) and electrode wear ratio (EWR) study on the powder mixed electrical discharge machining (PMEDM) of cobalt-bonded tungsten carbide (WC-Co) has been carried out. This type of cemented tungsten carbide was widely used as molding material of metal forming, forging, squeeze casting, and high pressure die casting. In the PMEDM process, the aluminum powder particle suspended in the dielectric fluid disperses and makes the discharging energy dispersion uniform; it displays multiple discharging effects within a single input pulse. This study was made only for the finishing stages and has been carried out taking into account the four processing parameters: discharge current, pulse on time, grain size, and concentration of aluminum powder particle for the machinability evaluation of MRR and EWR. The response surface methodology (RSM) has been used to plan and analyze the experiments. The experimental plan adopts the face-centered central composite design (CCD). This study highlights the development of mathematical models for investigating the influence of processing parameters on performance characteristics.

Sanjeev Kumar \& Rupinder Singh [12], although electrical discharge machining is essentially a material removal process, efforts have been made in the recent past to use it as a surface treatment method. An additive powder in the dielectric medium affects the sparking action and helps in improving the surface properties. It may melt at the high temperature of the plasma channel and alloy with the machined surface under appropriate machining conditions. Breakdown of the hydrocarbon dielectric contributes carbon to the plasma channel. In this paper, changes in surface properties of oil hardening non-shrinkable die steel after machining with manganese powder suspended in kerosene dielectric medium have been investigated. Results show improvement in micro hardness by $73 \%$, and no micro cracks on the machined surface. X-ray diffraction analysis of the machined surfaces reveals the transfer of manganese and carbon from the plasma channel in the form of manganese carbide. Quantitative analysis of chemical composition by optical emission spectrometer confirms significant increase in the percentages of manganese and carbon.

\section{Principle of Powder Mixed EDM}

The schematic of a PMEDM machine tool is shown in Figure

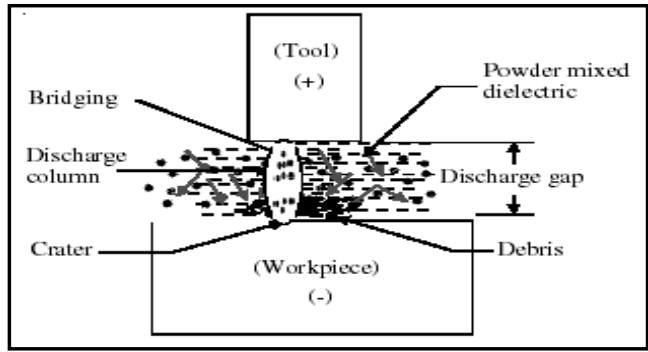

Figure 1.2: Principle of PMEDM Process

In this process, the material in powder form is mixed into the dielectric fluid either in the same tank or in a separate tank. When a voltage of $80-320 \mathrm{~V}$ is applied to both the electrodes, an electric field in the range 105 to $107 \mathrm{~V} / \mathrm{m}$ is created. The spark gap is filled up with additive particles, and the gap distance between tool and the work piece increases from $25 \mu \mathrm{m}$ to $50 \mu \mathrm{m}$ to many times larger. The powder particles get energized and behave in a zig-zag

\section{Volume 4 Issue 12, December 2015}




\section{International Journal of Science and Research (IJSR) \\ ISSN (Online): 2319-7064 \\ Index Copernicus Value (2013): 6.14 | Impact Factor (2014): 5.611}

fashion. The grains come close to each other under the sparking area and gather in clusters. Under the influence of electric forces, the powder particles arrange themselves in the form of chains at different places under the sparking area. The chain formation helps in bridging the gap between both the electrodes. Due to the bridging effect, the gap voltage and insulating strength of the dielectric fluid decreases. The easy short-circuit takes place, which causes early explosion in the gap. As a result, the ,series discharge ${ }^{\text {ee }}$ starts under the electrode area. Due to the increase in the frequency of discharging, the faster sparking within a discharge takes place, which causes faster erosion from the work piece surface. At the same time, the added powder modifies the plasma channel. The plasma channel gets enlarged and the electric density decreases; hence, sparking is uniformly distributed among the powder particles. As a result, even and more uniform distribution of the discharge takes place, which causes uniform erosion (shallow craters) on the work piece. This results in improvement in surface finish.

\section{Parameter of Study}

The various input parameters and output parameters (response variables) selected for the experimentation are as follows:

1) Input parameters

a) Discharge current (Ip)

b) Spark on time ( Ton)

c) Spark off time (Toff)

d) Gap voltage (volt)

e) Concentration of dielectric (C)

2) Output parameters

a) Material removal rate(MRR)

b) Surface Roughness ( $\mathrm{Ra}$ )

\section{Conclusion}

From this literature survey it will be concluded that the PMEDM (Powder Mixed Electric Discharge Machining) has significant effect on the material removal rate. With the addition of the powders in the dielectric, material removal rate has been increased to a great extent and the tool wear rate has been reduced. Silicon gives better results in terms of Material removal rate and tool wear rate. So, it is concluded and suggested to use silicon as an additive for PMEDM.

\section{References}

[1] H.K.Kansal , S.Singh and Pradeep kumar, "Effect of Silicon Powder Mixed EDM on Machining Rate of AISI D2 Die Steel", Journal of Manufacturing Processes Vol. 9/No. 12007.

[2] M. Kiyak and O. Cakir, "Examination of machining parameters on surface roughness in EDM of tool steel", Journal of Materials Processing Technology 191 (2007) 141-144.

[3] Anirban Bhattacharya \& Ajay Batish \& Gurmail Singh $\& V$. K. Singla, Optimal parameter settings for rough and finish machining of die steels in powder-mixed EDM, Int J Adv Manuf Technol (2012) 61:537-548.

[4] Katsushi Furutani \& Hiromichi Sato \& Masayuki
Suzuki, Influence of electrical conditions on performance of electrical discharge machining with powder suspended in working oil for titanium carbide deposition process; Int J Adv Manuf Technol (2009) 40:1093-1101.

[5] Paulo Peças \& Elsa Henriques, Effect of the powder concentration and dielectric flow in the surface morphology in electrical discharge machining with powder-mixed dielectric (PMD-EDM), Int J Adv Manuf Technol (2008) 37:1120-1132.

[6] Promod K. Patowari \& Partha Saha \& Prasanta K. Mishra, Taguchi analysis of surface modification technique using W-Cu powder metallurgy sintered tools in EDM and characterization of the deposited layer; Int J Adv Manuf Technol (2011) 54:593-604.

[7] KEESAM SHIN, DONGSUP CHUNG, and SUNGHAK LEE, „The Effect of Consolidation Temperature on Microstructure and Mechanical Properties in Powder Metallurgy-Processed 2XXX Aluminum Alloy Composites Reinforced with SiC Particulates; Center for Advanced Aerospace Materials, Pohang University of Science and Technology, Pohang, 790- 784 Korea.

[8] Y.-F. Tzeng and C.-Y. Lee, Effects of Powder Characteristics on Electro discharge Machining Efficiency; Int J Adv Manuf Technol (2001) 17:586592.

[9] Han-Ming Chow, Lieh-Dai Yang, Ching-Tien Lin, Yuan-Feng Chen, "The use of SiC powder in water as dielectric for micro-slit EDM machining", journal of materials processing technology 195 ( $\left.\begin{array}{lllllll}2 & 0 & 0 & 8\end{array}\right)$ 160170.

[10] Farhad Kolahan Mohammad Bironro, G.H., 2007. A HEURISTIC ALGORITHM FOR THE OPTIMAZATION OF POWDER-MIXED EDM PARAMETERS FOR TI-CO ALLOYS, Proceedings of the Third International Conference on Modeling, Simulation and Applied Optimization ,Sharjah, U.A.E January 20-22 2009.

[11]Kuang-Yuan Kung \& Jenn-Tsong Horng \& Ko-Ta Chiang, "Material removal rate and electrode wear ratio study on the powder mixed electrical discharge machining of cobalt-bonded tungsten carbide", Int J Adv Manuf Technol (2009) 40:95-104

[12] Sanjeev Kumar \& Rupinder Singh, "Investigating surface properties of OHNS die steel after electrical discharge machining with manganese powder mixed in the dielectric", Int J Adv Manuf Technol (2010) 50:625-633. 